\title{
Endometrial profilin 1: A key player in embryo- endometrial crosstalk
}

\author{
Chang-Jin Lee ${ }^{1, *}$, Seon-Hwa Hong,**, Min-Ji Yoon', Kyung-Ah Lee', Jung-Jae Ko', Hwa Seon Koo², Jee Hyun Kim², \\ Dong Hee Choi ${ }^{2}$, Hwang Kwon ${ }^{2}$, Youn-Jung Kang ${ }^{1,2,3}$ \\ 'Department of Biomedical Science, School of Life Science, ${ }^{2} \mathrm{CHA}$ Fertility Center Bundang, ${ }^{3}$ Department of Biochemistry, School of Medicine, CHA \\ University, Seongnam, Korea
}

\begin{abstract}
Objective: Despite extensive research on implantation failure, little is known about the molecular mechanisms underlying the crosstalk between the embryo and the maternal endometrium, which is critical for successful pregnancy. Profilin 1 (PFN1), which is expressed both in the embryo and in the endometrial epithelium, acts as a potent regulator of actin polymerization and the cytoskeletal network. In this study, we identified the specific role of endometrial PFN1 during embryo implantation.

Methods: Morphological alterations depending on the status of PFN1 expression were assessed in PFN1-depleted or control cells grown on Matrigel-coated cover glass. Day-5 mouse embryos were cocultured with Ishikawa cells. Comparisons of the rates of F-actin formation and embryo attachment were performed by measuring the stability of the attached embryo onto PFN1-depleted or control cells.

Results: Depletion of PFN1 in endometrial epithelial cells induced a significant reduction in cell-cell adhesion displaying less formation of colonies and a more circular cell shape. Mouse embryos co-cultured with PFN1-depleted cells failed to form actin cytoskeletal networks, whereas more F-actin formation in the direction of surrounding PFN1-intact endometrial epithelial cells was detected. Furthermore, significantly lower embryo attachment stability was observed in PFN1-depleted cells than in control cells. This may have been due to reduced endometrial receptivity caused by impaired actin cytoskeletal networks associated with PFN1 deficiency.

Conclusion: These observations definitively demonstrate an important role of PFN1 in mediating cell-cell adhesion during the initial stage of embryo implantation and suggest a potential therapeutic target or novel biomarker for patients suffering from implantation failure.
\end{abstract}

Keywords: Actin cytoskeletal network; Cell-cell adhesion; Embryo attachment; Embryo implantation; Profilin 1

Received: December 5, 2019 · Revised: December 9, 2019. Accepted: December 14, 2019 Corresponding author:Youn-Jung Kang

Department of Biochemistry, School of Medicine, CHA University, 43 Beolmal-ro 30beon-gil, Bundang-gu, Seongnam 13503, Korea

Tel: +82-31-881-7174 Fax:+82-31-881-7069 E-mail:yjkang@cha.ac.kr Co-Corresponding author: Dong Hee Choi

CHA Fertility Center Bundang, School of Medicine, CHA University, 43 Beolmalro 30beon-gil, Bundang-gu, Seongnam 13503, Korea

Tel: +82-31-727-8703 Fax:+82-31-881-7069 E-mail:dhchoimc@chamc.co.kr Co-Corresponding author: Hwang Kwon

CHA Fertility Center Bundang, School of Medicine, CHA University, 43 Beolmalro 30beon-gil, Bundang-gu, Seongnam 13503, Korea

Tel:+82-31-727-8706 Fax:+82-31-881-7069 E-mail:hkwon@chamc.co.kr

*These authors contributed equally to this work.

**This work was supported by funding from a National Research Foundation of Korea (NRF) grant funded by the Korean government (No. 2018R1C1B6003) to YJK.

This is an Open Access article distributed under the terms of the Creative Commons Attribution Non-Commercial License (http://creativecommons.org/licenses/by-nc/4.0/) which permits unrestricted non-commercial use, distribution, and reproduction in any medium, provided the original work is properly cited.

\section{Introduction}

Implantation failure is a major problem in assisted reproductive technology, with a large number of embryos underwent in vitro fertilization (IVF) failing to reach successful pregnancy [1,2]. Prior to embryo implantation, the endometrium undergoes dynamic changes induced by ovarian steroid hormones to produce a period of uterine receptivity referred to as the window of implantation [3-5]. This lasts from day 20 to day 24 of the menstrual cycle in humans [6]. After the endometrium becomes receptive and the embryo reaches the blastocyst stage, effective maternal-conceptus interaction must be initiated for successful implantation [7]. Failure of more than three IVF cycles in which reasonably good embryos were transferred is considered repeated implantation failure (RIF), which occurs in 15\%-20\% of infertile couples [8]. In patients with RIF, embryo transfer com- 
monly leads to only detection of human chorionic gonadotropin (hCG) or to no detectable status at all, meaning that embryo loss occurs at a very early stage of implantation [9]. Among the factors that attribute to RIF; decreased endometrial receptivity, defective embryos, and unsynchronized maternal-conceptus crosstalk; defects in endometrial receptivity account for approximately one-half of implantation failures in women suffering from RIF after embryo transfer $[1,9]$. However, little is known about the molecular mechanisms underlying the establishment of endometrial receptivity, in particular regarding the successful initial dialogue between the embryo and the maternal endometrium.

The actin cytoskeleton undergoes highly dynamic structural changes during cell migration, proliferation, and cell-cell adhesion [10]. When the embryo makes initial contact with the maternal endometrium at an early stage of implantation, cytoskeletal remodeling and actin polymerization play a crucial role [11]. It has been previously reported that aberrant actin reorganization impairs the development of mouse embryos produced by IVF during the pre-implantation stage [12]. Members of the profilin family, comprising four isoforms, have been identified as actin binding proteins that are essential for actin polymerization and cytoskeleton organization [13]. Profilin 1 (PFN1) is the most widely-understood protein in the profilin family, and it is reportedly expressed both in the endometrium and in the embryo [14-16]. The loss of PFN1 has been reported to induce the junctional delocalization of E-cadherin with a significant reduction in cell-cell and cell-extracellular matrix adhesion; additionally, it has been shown to elevate cell proliferation in human mammary epithelial cells. In contrast, PFN1 overexpression caused G1 cell cycle arrest as well as the inhibition of cell proliferation and tumor growth in human breast cancer cells $[17,18]$. However, the specific role of PFN1 in embryo attachment during the initial stage of implantation remains unknown. Therefore, in this study, we first examined the functional roles of PFN1 during the early stage of the embryo-endometrial interaction that mediates embryo attachment during implantation.

\section{Methods}

\section{Cell culture}

Ishikawa cells (a well-differentiated human endometrial adenocarcinoma line) obtained from American Type Cell Culture (Manassas, VA, USA) were maintained in a Dulbecco's Modified Eagle Medium/ F12 medium (Gibco, Grand Island, NY, USA) supplemented with 10\% fetal bovine serum (Gibco), $100 \mathrm{U} / \mathrm{mL}$ penicillin (Gibco), $100 \mathrm{mg} / \mathrm{mL}$ streptomycin (Gibco), and $2 \mathrm{mM} \mathrm{L-glutamine} \mathrm{(Gibco).} \mathrm{Cells} \mathrm{were}$ grown on Matrigel-coated cover glass (1:8 dilution, growth factor-reduced; Corning, Tewksbury, MA, USA) for further attachment assays.

\section{Plasmid transfection: lentivirus-mediated RNAi knockdown of PFN1}

To knock down PFN1 expression in Ishikawa cells, a lentiviral vector containing shRNA targeting PFN1 (shPFN1) and an empty vector (EV) were constructed with VSVG and d8.9 into L293 cells using the lipofectamine 2000 transfection protocol. The lentiviral vector was administered to Ishikawa cells for 24 hours, and cells were selected with puromycin. PFN1 expression after selection was verified by reversetranscription polymerase chain reaction (RT-PCR) and immunoblotting analyses.

\section{Mouse embryo collection and co-culture}

All experiments were conducted under a Home Office license, were in compliance with the Animal Act (1986) and had local ethical approval for the care and use of laboratory animals. C57BL/ 6 strain were maintained in strict accordance with the policies of the CHA University Institutional Animal Care and Use Committee (No. 190126). Female mice (6-8 weeks) were superovulated with $10 \mathrm{IU}$ of pregnant mare serum gonadotropin (Daesung Microbiological Labs, Seoul, Korea), and ovulation was synchronized with 5 IU of hCG (Sigma-Aldrich, St. Louis, MO, USA) 46-48 hours later, with both medications administered by intraperitoneal injection. Females were placed singly with males of the same strain overnight. The presence of a vaginal plug the following morning (day 1 of pregnancy) was used as an indicator of successful mating. Pregnant mice were killed on day 1 , 48 hours after hCG injection. One-cell embryos were obtained from the oviduct using a 30-G dissecting needle and a 1-mL syringe to tear open the ampulla of the oviduct and release the embryos. Cumulus cells around the one-cell embryos were washed with $0.1 \%$ hyaluronidase at $37^{\circ} \mathrm{C}$ for $5-10$ minutes and dissociated by gentle pipetting with glass pipettes. Collected embryos were washed with M2 medium (Sigma-Aldrich) supplemented with $4 \mathrm{mg} / \mathrm{mL}$ bovine serum albumin washed in potassium simplex optimized medium (KSOM; Millipore, Burlington, MA, USA) and cultured in a 20- $\mu \mathrm{L}$ drop of KSOM covered with mineral oil at $5 \% \mathrm{CO}_{2}$ and $37^{\circ} \mathrm{C}$ until the blastocyst stage. Only expanded blastocysts with clearly observable inner cell masses and trophectoderms on day 5 were included in the study.

\section{In vitro model for embryo implantation: assessment of the stability of embryo attachment}

Day-5 mouse embryos were transferred onto EV- or shPFN1-transduced Ishikawa cells in independent wells of a 24-well plate. Multiple observations of the stability of embryo attachment were performed to identify the distinct stages of attachment between 12 hours and 48 hours of co-culture, as previous reported [19,20]. A standardized plate movement protocol was applied to assess the stability of embryo attachment. The plate was tapped three times laterally and or- 
thogonally to detect unattached embryos. Five stages of attachment were defined and used as a measurement scale, as previously described [19,21].

\section{RNA isolation and PCR}

Total RNA was extracted from Ishikawa cells using TRlzol reagent (Ambion, Austin, TX, USA). A total of $1 \mu \mathrm{g}$ of RNA was used to synthesize complementary DNA using superscript IV reverse transcriptase (Invitrogen, Carlsbad, CA, USA) and oligo-dT primer. Using one-tenth of the volume of the complementary DNA, gene expression was quantitatively analyzed using an RT-PCR machine (Bio-Rad, Hercules, CA, USA). Amplification was performed using AccuPower PreMix (Bioneer, Daejeon, Korea) and programmed with $40-45$ cycles as follows: denaturation at $95^{\circ} \mathrm{C}$ for 10 minutes, annealing at $58^{\circ} \mathrm{C}-60^{\circ} \mathrm{C}$ for 30 seconds, and extension at $72^{\circ} \mathrm{C}$ for 30 seconds. The PCR products were subjected to electrophoresis using a $2 \%$ agarose gel and visualized with G-box software (Syngene, Cambridge, UK). The gene expression levels of the genes of interest were normalized to that of $\beta$-actin using ImageJ software.

\section{Immunoblotting analysis}

Whole lysate protein was extracted from cells using a radioimmunoprecipitation assay buffer with protease and phosphatase inhibitors (Thermo Fisher Scientific, Waltham, MA, USA) according to the manufacturer's protocol. In this process, $15 \mu \mathrm{g}$ of protein was separated by sodium dodecyl sulfate-polyacrylamide gel electrophoresis and transferred to a $0.2-\mu m$-pore PVDF membrane (Millipore). The membrane was blocked with $5 \%$ bovine serum albumin/Tris-Buff- ered Saline, $0.1 \%$ Tween 20 for 1 hour and then incubated with specific primary antibody at $4^{\circ} \mathrm{C}$ overnight against anti-PFN1 (1:2000; Abcam, Cambridge, UK) and $\beta$-actin (1:2000, Abcam), followed by incubation with mouse or rabbit immunoglobulin G-horseradish peroxidase (1:3000, Abcam). Protein bands were visualized using a Lumi Femto solution (Dogen, Seoul, Korea).

\section{Immunofluorescence staining}

Immunofluorescence staining using antibodies against PFN1 (1:500, Abcam) and phalloidin (1:1000, Abcam) were performed as described previously [22].

\section{Statistical analysis}

Comparison groups were analyzed with the unpaired Student $t$-test for parametric distributions. For multiple comparisons, two-way analysis of variance was used, followed by the Sidak multiple comparison test. For all tests, a $p$-value of $<0.05$ was considered to indicate statistical significance $(p<0.05, p<0.01, p<0.001$, and $p<$ 0.0001).

\section{Results}

\section{Confirmation of PFN1 knockdown in endometrial epithelial cells}

PFN1 acts as a potent regulator of actin cytoskeletal remodeling in response to extracellular signals in epithelial cells [23]. Knockdown of PFN1 expression suppresses fibronectin-promoted cell proliferation and migration in gastric cancer cells [24]. However, the role of PFN1
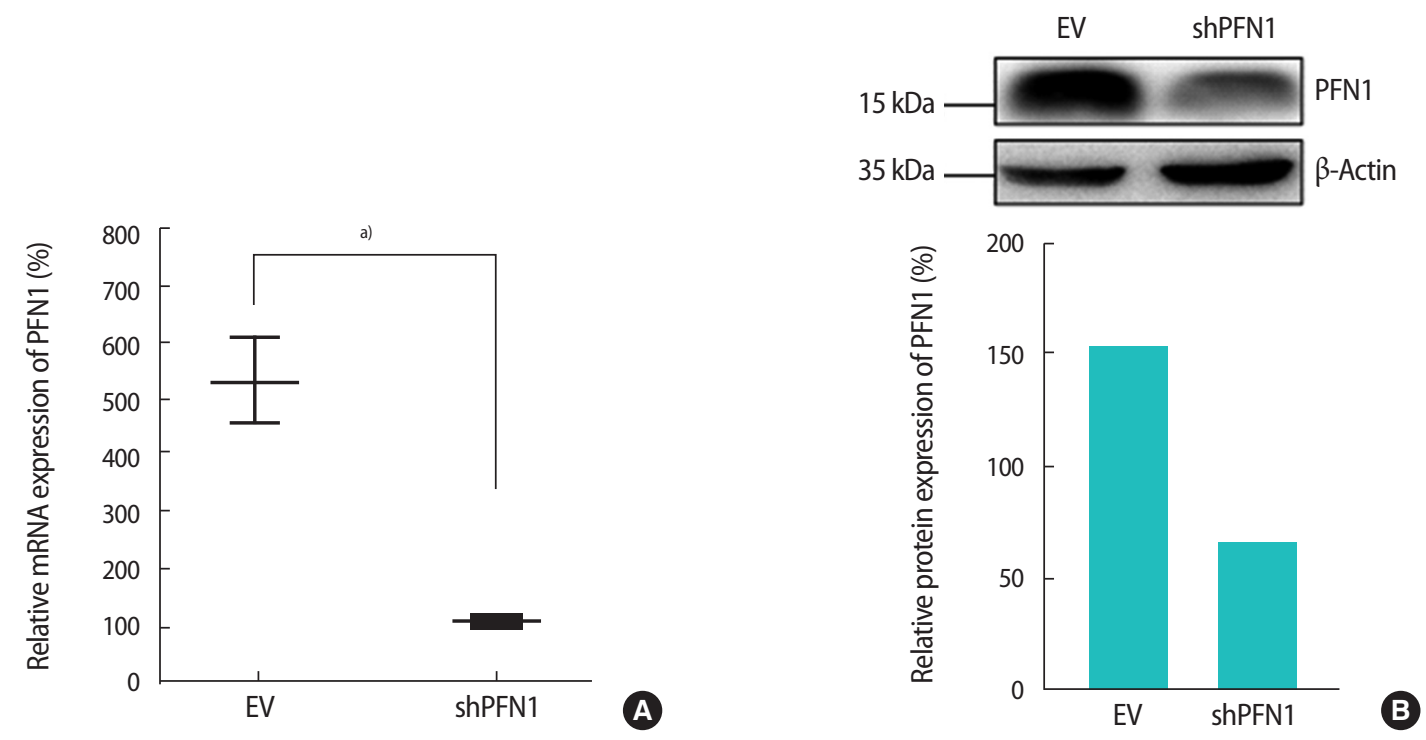

Figure 1. Reverse-transcription polymerase chain reaction (A) and immunoblotting analyses (B) were used to quantify the mRNA and protein expression of profilin 1 (PFN1) in shRNA targeting PFN1 (shPFN1)-transduced Ishikawa cells compared to empty vector (EV)-transduced cells. The densitometry of immunoblot bands shown in (B) was measured using Image J software. ${ }^{\text {a) }} 0.01<p<0.05$. 
in endometrial epithelial cells has not yet been examined. To address whether PFN1 in the endometrial epithelium plays an important role in mediating embryonic attachment at the initial stage of implantation, human endometrial epithelial cells (Ishikawa cells) were infected with a lentiviral vector expressing a construct targeting PFN1 shRNA (shPFN1). EV-transduced Ishikawa cells were used as controls across all experiments in this study. Knockdown of PFN1 expression was confirmed by RT-PCR and an immunoblotting assay, demonstrating approximately a six-fold reduction in mRNA and a 2.5-fold decrease in protein levels compared to control-vector-transduced control cells (Figure 1). Morphological examination of Ishikawa cells depending on the status of PFN1 expression revealed that PFN1-depleted Ishikawa cells showed a relatively low extent of spreading and displayed a more circular shape at the edge of cells compared to EVtransduced cells, which is consistent with previous reports showing that the actin cytoskeleton acts as a primary determinant of cell shape and motility [25]. Additionally, more clustering was observed in EV-transduced control cells than in the PFN1-depleted cells (Figure 2). Our data suggest that PFN1 plays a role in mediating cell-cell interactions by regulating the actin cytoskeletal network.

\section{Knockdown of endometrial PFN1 suppresses the actin cytoskeletal network between the embryo and the endometrium}

Both cell-cell and cell-extracellular matrix adhesions are facilitated by physical association with the actin cytoskeleton and efficient transmission of the intracellular signaling that promotes cellular adhesion and migration to establish cell-cell interaction [26]. To address
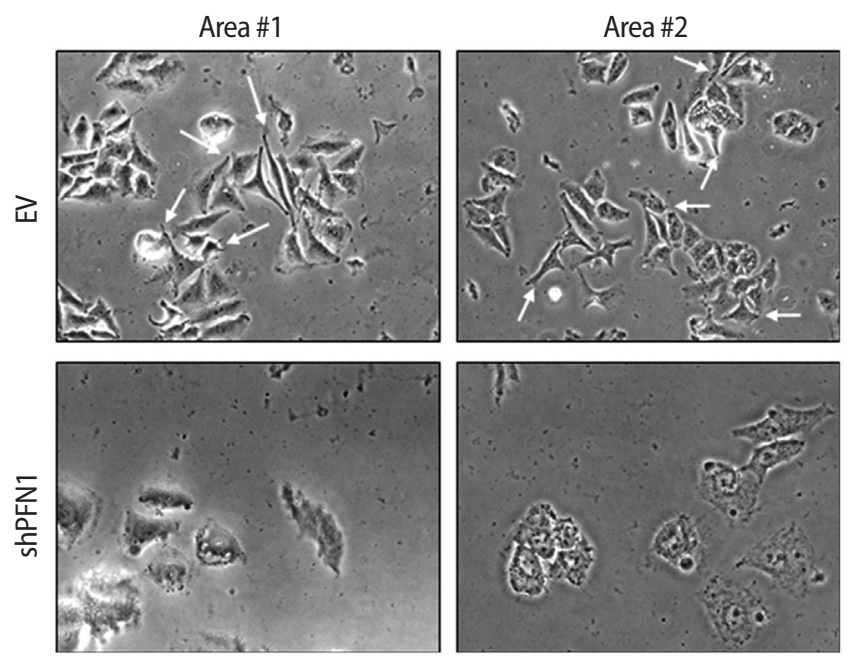

Figure 2. Morphological alterations in shRNA targeting profilin 1 (shPFN1) or empty-vector (EV)-transduced Ishikawa cells grown on Matrigel-coated cover glass. Cell spreading was assessed 48 hours after seeding. White arrows indicate the edges of spreading cells. whether this is applicable for the initial cell-cell interactions between the embryo and the maternal endometrium, embryonic F-actin formation at the attachment sites on shPFN1-transduced Ishikawa cells compared to EV-transduced control cells was assessed using phalloidin staining. For immunofluorescence analysis, day-5 mouse embryos were co-cultured with shPFN1- or EV-transduced Ishikawa cells. After 48 hours of co-culture, all the embryos used for F-actin formation analyses, were found to be stably attached to both shPFN1- and EV-transduced cells. Embryos attached to EV-transduced Ishikawa cells exhibited more F-actin spreading towards the Ishikawa cells, whereas very little phalloidin expression was detected surrounding the embryos which were attached to shPFN1-transduced cells (Figure 3). Interestingly, all embryos showed significantly higher phalloidin expression than the Ishikawa cells. This may have been due to the difference in depth on microscopy, as embryos in the upper phase express higher levels of phalloidin than Ishikawa cells in the lower phase.

\section{Knockdown of PFN1 in endometrial epithelial cells reduces the rate of embryo attachment at the initial stage of implantation}

Following the transduction of EV or shPFN1 in Ishikawa cells, a total of 1765 -day-old mouse embryos were transferred (one embryo per

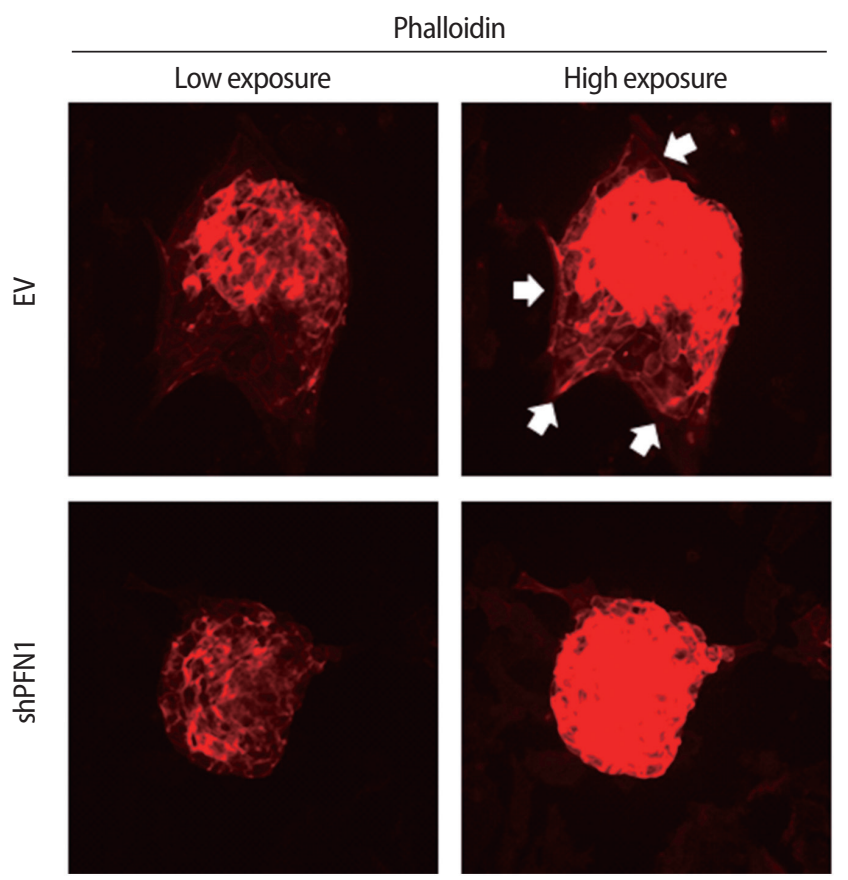

Figure 3. Immunofluorescence staining of phalloidin in mouse embryos co-cultured with empty vector (EV) or shRNA targeting profilin 1 (shPFN1)-transduced Ishikawa cells. White arrows point to embryonic F-actin spreading towards Ishikawa cells. 


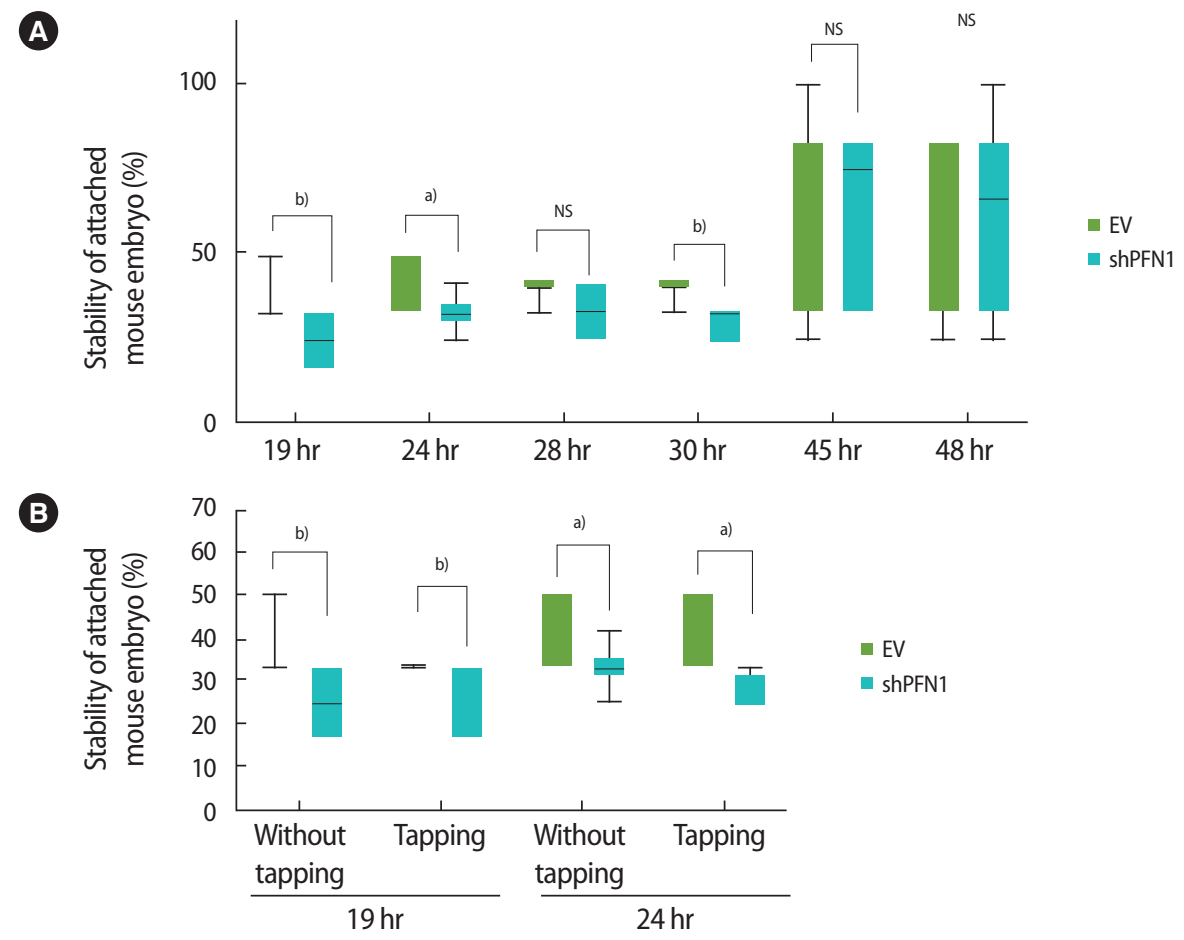

Figure 4. (A) Mean proportions (using 176 day-5 mouse embryos in total) of the stability of embryo attachment after $19,24,28,30,45$, and 48 hours of coculture to empty vector (EV)-transduced or shRNA targeting profilin 1 (shPFN1)-transduced Ishikawa cells. (B) Comparison of mean proportions of the stability of attached embryos at 19 hours and 24 hours with or without disturbance by tapping the plate. Values are presented as mean \pm standard error of the mean. NS, not significant. Statistically significant differences: ${ }^{\mathrm{a})} 0.01<p<0.05,{ }^{\mathrm{b})} 0.001<p<0.01$.

well) to confluent cells and were co-cultured for $19,24,28,30,45$, and 48 hours. The stability of the attached mouse embryo was measured according to the five-stage standard: 1 , floating; 2 , weakly attached but detached after tapping; 3 , weakly attached but stuck at the attachment site after tapping; 4 , stably attached; and 5 , stably attached and showing outgrowth $[19,21]$. After up to 30 hours of coculture, embryos transferred on control-vector-transduced Ishikawa cells were found to be significantly more stably attached than embryos cocultured with shPFN1-transduced cells, whereas no significant difference was observed at the late stage of co-culture (Figure 4A). Additionally, stably-attached mouse embryos were disturbed by tapping the plates. Interestingly, we found that embryos associated with EV-transduced Ishikawa cells remained more stable than those cocultured with PFN1-depleted cells (Figure 4B). However, after 48 hours of coculture, no significant difference was observed. This might implicate that endometrial PFN1 plays an important role in the early stage of embryo attachment.

\section{Discussion}

The data presented in this study revealed the importance of PFN1, a potent regulator of actin polymerization and cytoskeletal remodel- ing, in facilitating the initial attachment and adhesion between trophoblast cells of the embryo and epithelial cells of the maternal endometrium. This suggests that the PFN1 gene is a critical mediator of the initial stage of embryo implantation. Implantation is historically known to consist of three stages: apposition, adhesion, and invasion $[3,27,28]$. Both mouse and human embryos exhibit sequential events of embryo attachment beginning with weak initial attachment and subsequent more stable adhesion to the maternal endometrial epithelium [29]. During the attachment stage, appropriate crosstalk between the endometrium and the embryo is crucial for successful implantation, and abnormalities occurring during this process can be the cause of pregnancy failure $[3,27,30]$. Embryonic loss in patients suffering from RIF often occurs during this early stage of implantation [9]. Despite the extensive research aimed at overcoming implantation failure, especially with regard to embryonic loss at the attachment stage, no clear evidence-based therapeutic strategy currently exists.

PFN1, one of the main regulators of actin dynamics, is a key mediator of cell-cell adhesion in the formation of colonies and the promotion of cell spreading [31,32]. The characteristics observed in endometrial epithelial cells as shown in Figure 2 might be fundamentally applicable to the initial interaction or attachment between the em- 
bryo and the maternal endometrial epithelium. Similar to previous findings that showed a significant reduction of F-actin formation with depletion of PFN1 in alveolar epithelial cells [33], our data demonstrated that embryos attached to PFN1-depleted Ishikawa cells displayed reductions in F-actin and stress fiber formation by exhibiting decreased phalloidin expression compared to EV-transduced cells (Figure 3). This may imply that a reduced capacity of cytoskeletal networking between the embryo and the maternal endometrium caused by the absence of endometrial PFN1 impairs endometrial receptivity and decreases the chances of association between these two different types of cells. Additionally, within the cooperative regulation of vinculin and vasodilator-stimulated phosphoprotein (VASP), PFN1 is known to help maintain epithelial cell polarity [34], which is critical for the embryo to initiate attachment onto the maternal epithelium for successful implantation [27]. Depletion of PFN1 might have induced the loss of polarity in Ishikawa cells and subsequently caused the reduction in the rate of embryo implantation at an early point. Furthermore, PFN1 (both nuclear and cytoplasmic) is known to play key roles in oocyte maturation, fertilization, and embryonic development [35]. PFN1 is also detected in serum and conditioned media $[36,37]$. Although its extracellular function is not well understood, extracellular PFN1 has been reported to be involved in the decidualization of human endometrial stromal cells by acting as an extravillous trophoblast-secreted factor [38], suggesting the importance of a future study examining the impact of extracellular PFN1 on changes in expression at the cellular level in endometrial epithelial cells and improvement of the embryo-endometrial networking environment. However, it was not clear whether proper actin polymerization through intact PFN1 facilitates embryonic attachment to the endometrium. To address this question, we evaluated the role of PFN1 in embryo attachment by measuring the stability of attached embryos at serial time points using a previously-established in vitro model of embryo implantation $[19,20]$. Our data showed that attachment was delayed in embryos transferred onto PFN1-depleted Ishikawa cells compared to those cocultured with control cells at early points (19 to 30 hours), even though all embryos were stably attached at the late stage of attachment (by 48 hours) (Figure 4). Liang et al. [39] reported that the knockdown of TAGLN2, an actin-binding protein, in trophoblast cells remarkably reduced F-actin formation and impaired trophoblast adhesion and invasion, supporting the importance of actin polymerization in successful embryo implantation, especially during the initial stages.

Overall, this study identified that PFN1 plays an important role in actin cytoskeleton dynamics, which are critical for mediating the cellcell adhesion between the blastocyst and the maternal endometrial epithelium required for successful initial embryo attachment during implantation. Quantification of the stability of the attached embryo revealed that an impaired cytoskeletal environment associated with PFN1 deficiency in the endometrial epithelium significantly reduced the rate of embryo attachment during the early stage of implantation compared to embryos cocultured with PFN1-intact endometrial epithelial cells. Future studies addressing the relevance of the status of endometrial PFN1 with infertility caused by implantation failure may further aid in the identification of new therapeutic targets or novel biomarkers for patients suffering from RIF.

\section{Conflict of interest}

Hwa Seon Koo has been a managing editor of Journal of Clinical and Experimental Reproductive Medicine since 2018; however, she was not involved in the peer reviewer selection, evaluation, or decision process of this article. No other potential conflicts of interest relevant to this article were reported.

\section{ORCID}

Chang-Jin Lee $\quad$ https://orcid.org/0000-0003-4629-8052

Seon-Hwa Hong https://orcid.org/0000-0002-0663-1781

Min-Ji Yoon https://orcid.org/0000-0002-8098-7073

Kyung-Ah Lee https://orcid.org/0000-0001-6166-5012

Jung-Jae Ko https://orcid.org/0000-0002-5361-5784

Hwa Seon Koo https://orcid.org/0000-0003-2783-5668

Jee Hyun Kim https://orcid.org/0000-0002-2584-108X

Dong Hee Choi https://orcid.org/0000-0001-7242-8987

Hwang Kwon https://orcid.org/0000-0003-0775-2881

Youn-Jung Kang https://orcid.org/0000-0002-0771-9515

\section{Author contributions}

Conceptualization: YJK. Data curation: YJK, CJL, SHH, HSK. Formal analysis: YJK, CJL, SHH, KAL, JJK. Funding acquisition: YJK. Methodology: MJY, JHK. Project administration: YJK, DHC, HK. Visualization: CJL, SHH. Writing-original drafting, review \& editing: YJK.

\section{References}

1. Bashiri A, Halper KI, Orvieto R. Recurrent Implantation Failureupdate overview on etiology, diagnosis, treatment and future directions. Reprod Biol Endocrinol 2018;16:121.

2. Simon A, Laufer N. Assessment and treatment of repeated implantation failure (RIF). J Assist Reprod Genet 2012;29:1227-39.

3. Dey SK, Lim H, Das SK, Reese J, Paria BC, Daikoku T, et al. Molecular cues to implantation. Endocr Rev 2004;25:341-73.

4. Psychoyos A. Hormonal control of ovoimplantation. Vitam Horm 
1973;31:201-56.

5. Tabibzadeh S. Molecular control of the implantation window. Hum Reprod Update 1998;4:465-71.

6. Achache H, Revel A. Endometrial receptivity markers, the journey to successful embryo implantation. Hum Reprod Update 2006; 12:731-46.

7. Norwitz ER. Defective implantation and placentation: laying the blueprint for pregnancy complications. Reprod Biomed Online 2006;13:591-9.

8. Margalioth EJ, Ben-Chetrit A, Gal M, Eldar-Geva T. Investigation and treatment of repeated implantation failure following IVF-ET. Hum Reprod 2006;21:3036-43.

9. Koot YE, Boomsma CM, Eijkemans MJ, Lentjes EG, Macklon NS. Recurrent pre-clinical pregnancy loss is unlikely to be a'cause' of unexplained infertility. Hum Reprod 2011;26:2636-41.

10. Tang DD, Gerlach BD. The roles and regulation of the actin cytoskeleton, intermediate filaments and microtubules in smooth muscle cell migration. Respir Res 2017;18:54.

11. Bazer FW, Wu G, Spencer TE, Johnson GA, Burghardt RC, Bayless K. Novel pathways for implantation and establishment and maintenance of pregnancy in mammals. Mol Hum Reprod 2010; 16:135-52.

12. Tan K, An L, Wang SM, Wang XD, Zhang ZN, Miao K, et al. Actin disorganization plays a vital role in impaired embryonic development of in vitro-produced mouse preimplantation embryos. PLoS One 2015;10:e0130382.

13. Alkam D, Feldman EZ, Singh A, Kiaei M. Profilin1 biology and its mutation, actin(g) in disease. Cell Mol Life Sci 2017;74:967-81.

14. Berglund L, Bjorling E, Oksvold P, Fagerberg L, Asplund A, Szigyarto $C A$, et al. A genecentric human protein atlas for expression profiles based on antibodies. Mol Cell Proteomics 2008;7:201927.

15. Thul PJ, Akesson L, Wiking M, Mahdessian D, Geladaki A, Ait Blal $\mathrm{H}$, et al. A subcellular map of the human proteome. Science 2017;356:eaal3321.

16. Uhlen M, Fagerberg L, Hallstrom BM, Lindskog C, Oksvold P, Mardinoglu A, et al. Proteomics: tissue-based map of the human proteome. Science 2015;347:1260419.

17. Bae YH, Ding Z, Zou L, Wells A, Gertler F, Roy P. Loss of profilin-1 expression enhances breast cancer cell motility by Ena/VASP proteins. J Cell Physiol 2009;219:354-64.

18. Zou L, Ding Z, Roy P. Profilin-1 overexpression inhibits proliferation of MDA-MB-231 breast cancer cells partly through p27kip1 upregulation. J Cell Physiol 2010;223:623-9.

19. Kang YJ, Forbes K, Carver J, Aplin JD. The role of the osteopontinintegrin av $\beta 3$ interaction at implantation: functional analysis using three different in vitro models. Hum Reprod 2014;29:739-49.
20. Kang YJ, Lees M, Matthews LC, Kimber SJ, Forbes K, Aplin JD. MiR-145 suppresses embryo-epithelial juxtacrine communication at implantation by modulating maternal IGF1R. J Cell Sci 2015;128:804-14.

21. Carver J, Martin K, Spyropoulou I, Barlow D, Sargent I, Mardon H. An in-vitro model for stromal invasion during implantation of the human blastocyst. Hum Reprod 2003;18:283-90.

22. Kang YJ, Balter B, Csizmadia E, Haas B, Sharma H, Bronson R, et al. Contribution of classical end-joining to PTEN inactivation in p53-mediated glioblastoma formation and drug-resistant survival. Nat Commun 2017;8:14013.

23. Ding Z, Roy P. Profilin-1 versus profilin-2: two faces of the same coin? Breast Cancer Res 2013;15:311.

24. Cheng YJ, Zhu ZX, Zhou JS, Hu ZQ, Zhang JP, Cai QP, et al. Silencing profilin-1 inhibits gastric cancer progression via integrin $\beta 1 /$ focal adhesion kinase pathway modulation. World J Gastroenterol 2015;21:2323-35.

25. Suetsugu S, Takenawa T. Regulation of cortical actin networks in cell migration. Int Rev Cytol 2003;229:245-86.

26. Bachir Al, Horwitz AR, Nelson WJ, Bianchini JM. Actin-based adhesion modules mediate cell interactions with the extracellular matrix and neighboring cells. Cold Spring Harb Perspect Biol 2017;9:a023234.

27. Aplin JD, Ruane PT. Embryo-epithelium interactions during implantation at a glance. J Cell Sci 2017;130:15-22.

28. Nimbkar-Joshi S, Rosario G, Katkam RR, Manjramkar DD, Metkari SM, Puri CP, et al. Embryo-induced alterations in the molecular phenotype of primate endometrium. J Reprod Immunol 2009; 83:65-71.

29. Singh H, Aplin JD. Adhesion molecules in endometrial epithelium: tissue integrity and embryo implantation. J Anat 2009;215: 3-13.

30. Kim SM, Kim JS. A review of mechanisms of implantation. Dev Reprod 2017;21:351-9.

31. Ding Z, Lambrechts A, Parepally M, Roy P. Silencing profilin-1 inhibits endothelial cell proliferation, migration and cord morphogenesis. J Cell Sci 2006;119(Pt 19):4127-37.

32. Zou L, Hazan R, Roy P. Profilin-1 overexpression restores adherens junctions in MDA-MB-231 breast cancer cells in R-cadherindependent manner. Cell Motil Cytoskeleton 2009;66:1048-56.

33. Bitko V, Oldenburg A, Garmon NE, Barik S. Profilin is required for viral morphogenesis, syncytium formation, and cell-specific stress fiber induction by respiratory syncytial virus. BMC Microbiol 2003;3:9.

34. Quinlan MP. Vinculin, VASP, and profilin are coordinately regulated during actin remodeling in epithelial cells, which requires de novo protein synthesis and protein kinase signal transduction 
pathways. J Cell Physiol 2004;200:277-90.

35. Rawe VY, Payne C, Schatten G. Profilin and actin-related proteins regulate microfilament dynamics during early mammalian embryogenesis. Hum Reprod 2006;21:1143-53.

36. Caglayan E, Romeo GR, Kappert K, Odenthal M, Sudkamp M, Body SC, et al. Profilin-1 is expressed in human atherosclerotic plaques and induces atherogenic effects on vascular smooth muscle cells. PLoS One 2010;5:e13608.

37. Thery C, Boussac M, Veron P, Ricciardi-Castagnoli P, Raposo G,
Garin J, et al. Proteomic analysis of dendritic cell-derived exosomes: a secreted subcellular compartment distinct from apoptotic vesicles. J Immunol 2001;166:7309-18.

38. Menkhorst EM, Van Sinderen ML, Rainczuk K, Cuman C, Winship A, Dimitriadis E. Invasive trophoblast promote stromal fibroblast decidualization via Profilin 1 and ALOX5. Sci Rep 2017;7:8690.

39. Liang X, Jin Y, Wang H, Meng X, Tan Z, Huang T, et al. Transgelin 2 is required for embryo implantation by promoting actin polymerization. FASEB J 2019;33:5667-75. 